\title{
Dose tracking assessment for image-guided radiotherapy of the prostate bed and the impact on clinical workflow
}

\author{
Lucia Clara Orlandini ${ }^{1,2}$, Marianna Coppola ${ }^{1}$, Christian Fulcheri ${ }^{3}$, Luna Cernusco ${ }^{1}$, Pei Wang ${ }^{2^{*}}$ and Luca Cionini ${ }^{1}$
}

\begin{abstract}
Background: The cumulative dose was compared with the planned dose among fourteen patients undergoing image-guided, intensity-modulated radiotherapy of the prostate bed. Moreover, we investigated the feasibility of adding dose tracking to the routine workflow for radiotherapy.

Methods: Daily cone beam computed tomography was conducted for image-guided radiotherapy, and weekly cumulative delivered doses were calculated for dose tracking. Deformable image registration was applied to map weekly dose distributions to the original treatment plan and to create a cumulative dose distribution. The dose-volume histogram (DVH) cut-off points for the rectum and bladder and the planning target volume (PTV), were used to compare the planned and cumulative delivered doses. The additional time required by the departmental staff to complete these duties was recorded.

Results: The PTV coverage of the delivered treatment did not satisfy the expected goal for three patients (V98\% > 98\%). In another three patients, the DVH cut-off point for the bladder was higher than the limits, while for the rectum, treatment was as expected in all cases (two patients failed both their bladder constraints and the PTV coverage). Overall, four patients did not satisfy one or more criteria at the end of their treatment.

Conclusions: A well-defined strategy for dose tracking assessment is feasible, would have minimal impact on the workload of a radiotherapy department, and may offer objective information to support radiation oncologists in making decisions about adaptive procedures.
\end{abstract}

Keywords: Radiotherapy, Image-guided radiotherapy, Intensi-modulated radiation therapy, Prostate cancer

\section{Background}

Intensity-modulated radiotherapy (IMRT) techniques are now extensively used for radical treatments in patients with localized and locally advanced prostate cancer and in post-prostatectomy settings [1-3]. The main dosimetric advantage of IMRT is that high conformal dose distribution can be obtained, particularly in the presence of a concavely shaped target. This enables the safer delivery of higher doses to the target and better sparing of organs at risk (OARs), namely the rectum and bladder. However, the modifications or displacements of target volumes and OARs, relative to the radiation beam frequently occur

\footnotetext{
*Correspondence: dengwangpei@163.com

${ }^{2}$ Department of Radiation Oncology, Sichuan Cancer Hospital, No.55, the 4th

Section, Renmin South Road, Chengdu, China

Full list of author information is available at the end of the article
}

because of patient positioning and different filling and pressure effects from OARs; these can result in significant dosimetric changes because of the sharp dose gradients between the target and normal tissue [4-7]. Unfortunately, such anatomical modifications may cause under-dosing of target organs and/or over-dosing of OARs.

Image-guided radiotherapy (IGRT) is commonly used to reduce setup errors in patient positioning and in the inter-fraction organ motion [8, 9]. Typically, the correction parameter involves moving the treatment table to re-position the shifted target point to the isocenter of the treatment device. Such a target-point correction is a widespread strategy in IGRT, and it has proven to be superior for treatment of tumour sites with less pronounced deformations. However, this technique does 
not correct the anatomical deformations, such as volume changes that result from modifications or filling differences in OARs, or variations in the planning target volume (PTV) that occur during the course of treatment. Previous studies have argued regarding the use of adaptive radiotherapy and how it can be applied to manage inter-fraction motion [10,11] and have proposed offline strategies based on geometric and dosimetric feedback $[12,13]$. To date, the complexity of the dosimetric adaptive radiotherapy means that it is not a common part of routine clinical practice.

We investigated how inter-fractional variations in patient anatomy affected the difference between planned and delivered doses in patients undergoing adjuvant IGRT-IMRT for prostate cancer. A detailed analysis was performed among fourteen patients undergoing prostate radiotherapy. The additional time required by a multidisciplinary group (therapists, radiation oncologists and medical physicists) to complete these duties was recorded to assess whether this working schedule was practicable in daily practice. We calculated the delivered dose weekly and compared the planned dose with the cumulative delivered dose at the end of the treatment course. Dose-volume histogram (DVH) cut-off points for the rectum and bladder, as well as the DVH target coverage, were used for the comparison. The additional time burden was analysed for each health professional involved. We aimed to investigate, retrospectively, the effectiveness and feasibility of including dose tracking in the clinical radiotherapy workflow in order to manage an appropriately timed adaptive procedure.

\section{Methods}

\section{Patients selection and treatment planning optimisation}

Fourteen patients with prostate carcinoma undergoing an adjuvant prostate treatment and who received IGRTIMRT routinely were included in this study. Patients were treated according to our center's protocol, with prescribed dose of 70 Gy delivered in fractions of 2 Gy daily. The clinical target volume (CTV) included the prostatic lodge and was defined following the RTOG consensus guidelines for postoperative radiation therapy [14]. The PTV was obtained by adding a 5-mm margin to the CTV in every direction, except for the posterior direction where a 4-mm margin was used to limit the dose to the rectum. Computed Tomography (CT) images were acquired with patients in the supine position, with their feet fixed in a support, and a wedge placed under the knee to avoid pelvic rotation; a 3-mm slice width was used. Magnetic resonance (MR) images were acquired in the same position, immediately after the CT scan. Patients were instructed to maintain an empty rectum and a full bladder for the CT scans, MR scans, and treatment. 2D-CT and MR images were rigidly registered with a multi modalities image registration software (Mirada XD, Mirada Medical Ltd, Oxford, UK); T2-weighted turbo spin echo transverse MR sequence was used for the fusion. The contouring of the prostate, rectum, and bladder was defined on the fusion image set by an experienced radiation oncologist (RO).

All patients were treated with $6 \mathrm{MV}$ photon beams at seven equally spaced gantry angles. A Siemens Artiste linear accelerator (Siemens Medical Solution, Erlangen, Germany) equipped with a Megavoltage Cone Beam Computed Tomography (MV-CBCT) was used. The treatment planning was performed with a Pinnacle ${ }^{3 T M}$ Step-and-Shoot IMRT system ( $\mathrm{P}^{3} \mathrm{IMRT}$, Version 9.0, Philips Medical Division, Madison, WI), using direct machine parameter optimisation. CBCT performed daily with the $6 \mathrm{MV}$ photon beam of the linac was included in the treatment plan as an arc field $[15,16]$, and the daily imaging dose of the $8 \mathrm{MU}$ CBCT protocol was integrated into the prescribed dose [17]. In Table 1 the daily CBCT mean dose contribution to PTV mean dose is reported.

Plan optimisation was based on dose volume objectives for PTVs and on OAR constraints commonly used in clinical practice [18-20]; in Table 2 the adopted values are shown. We define Dx\% as the dose (in Gy) received by $\mathrm{x} \%$ of the volume, and $\mathrm{Vy}$ as the volume (in percentage) that receives y Gy. For the PTV, we defined $D_{\text {max }}$ as the dose received by $1 \mathrm{~cm}^{3}$ of the target volume. We aimed to achieve a final plan delivering the prescribed dose to at least $98 \%$ of the tumour volume, but no more than $107 \%$ of the prescribed dose to no more than $1 \%$ of the tumour volume, while ensuring that OAR doses remained as low as achievable.

To evaluate dose tracking, we used the Raystation Demo Version 4.5 Treatment Planning System (RaySearch Laboratories AB Stockholm, Sweden) with the machine used for treatment. The treatment planning delivered to the patient and approved by the RO carried out with Pinnacle was re-calculated with Raystation TPS on the reference CT images. This treatment planning was taken as the baseline for the treatment. The DVH of the planned treatment, with the resulting OAR cut-off points and percentage target coverage, was used to compare the differences between the cumulated delivered and approved doses.

\section{IGRT and retrospective dose tracking}

Patients underwent daily IGRT with a megavoltage CBCT (MV-CBCT) mounted on the treatment machine. The MV-CBCT used the linear accelerator as the

Table 1 Imaging contribution to the prescribed dose

\begin{tabular}{lcl}
\hline & IMRT & MV-CBCT \\
\hline MU & $548 \pm 84$ & 8 \\
Dose (cGy) & $193.68 \pm 0.48$ & $6.31 \pm 0.45$
\end{tabular}

MUs and corresponding doses of the IMRT and MV-CBCT are reported 
Table 2 Organs at risk dose-volume constraints and goal for the planning target volume (PTV) used for adjuvant prostate IMRT planning

\begin{tabular}{lll}
\hline Organ & Dose (Gy) & Volume (\%) \\
\hline Bladder & 50 & 65 \\
& 65 & 50 \\
Rectum & 70 & 35 \\
& 40 & 60 \\
& 50 & 50 \\
& 60 & 35 \\
Femoral Heads & 70 & 20 \\
& 30 & 50 \\
Small Bowell & 50 & 10 \\
& 15 & $120 \mathrm{cc}$ \\
PTV & 45 & $195 \mathrm{cc}$ \\
\hline
\end{tabular}

${ }^{\text {a }}$ Prescription Dose

All constraints are based on a schedule of $2 \mathrm{~Gy} /$ fraction

radiation source and a $40 \times 40 \mathrm{~cm}^{2}$ amorphous-silicon electronic portal imaging for online volumetric imaging. The technical specifications of the MVision system have been described elsewhere in the literature [21]. The protocol involved a $200^{\circ}$ gantry arc rotation from $270^{\circ}$ to $110^{\circ}$ of a $6-\mathrm{MV}$ beam around the isocenter with a source-to-axis distance of $100 \mathrm{~cm}$ and a source-to-image distance of $145 \mathrm{~cm}$. We acquired 200 two-dimensional projection images and combined them to reconstruct a volumetric CT image dataset with a field of view diameter of $27.4 \mathrm{~cm}$. A protocol of 8 monitor units was adopted for prostate imaging. The daily CBCT scans before treatment were matched with the original CT scans by the RO and were used clinically to align the relevant anatomic structures of the pelvis including rectum and bladder to the anatomy. The patient's isocenter was moved to the corrected position, as appropriate, and the daily displacement was recorded.

For each patient, the cumulative delivered dose was calculated weekly. A total of 7 CBCTs were included in the recalculation (assuming a reference $\mathrm{CBCT}$ for the entire week). To avoid discrepancies that were not strictly connected to the anatomical changes of the patient or their position, a first comparison was performed between the planned $\mathrm{CT}$ treatment and that calculated on day one of $\mathrm{CBCT}\left(\mathrm{CBCT}_{0}\right)$. Only patients for whom the difference (cut-off points for OARs and target coverage) between the dose calculated on the planning $\mathrm{CT}$ and the dose on $\mathrm{CBCT}_{0}$ was less than $1 \%$ were included in the study.

Rigid registration was done to take into account the shift applied to the treatment isocenter during online correction. A deformable registration, based on biomechanical modelling and finite element analysis, was then performed [22-25] with Raystation platform also used for dose tracking. The obtained deformation map was used to map the target and the OARs of the weekly CBCT to the original planning CT; a new geometry was then achieved for the selected structures. The same experienced RO who performed the daily matching also assessed the correctness of the propagated regions of interest on each slice of the $\mathrm{CBCT}$, and adjusted them if necessary. A second deformable registration was then calculated based on these verified contours, and a final deformation map was obtained; the contours defined in both the CT images drove the deformable registration. After computing dose in the CBCT datasets, they were deformed back to the planning scan and accumulated for DVH analysis. The process of dose recalculation and dose accumulation in Raystation, as well as the dose deformation algorithms, have been discussed in detail in literature [26, 27].

The most significant rectal and bladder cut-off points for tracking during treatment were identified by the RO for each patient, and a comparison was performed between the accumulated delivered doses and the planned treatment doses. For each plan, the most significant DVH cut-off, among those reported in Table 1, was the one most likely not to be met at the end of the treatment course. The tolerance was given by respect to these OAR limits. For comparison of the PTV coverage, we used the $D_{98 \%}$ and $D_{\max }$ value: for the $D_{98 \%}$ parameter, we accepted a difference of $1.7 \mathrm{~Gy}$ (2.5\% difference) between the delivered and prescribed dose; while for the $D_{\max }$ value, we accepted a 3 Gy difference.

Planned and delivered doses were compared based on the relative percentage dose differences (\%diff D) as follows: \%diff $\mathrm{D}=100 \times\left(\mathrm{D}_{98 \% \mathrm{D}^{-}}-\mathrm{D}_{98 \% \mathrm{P}}\right) \mathrm{D}_{98 \% \mathrm{p}}$; where $\mathrm{D}_{98 \% \mathrm{P}}$ and $\mathrm{D}_{98 \% \mathrm{D}}$, represent the dose received by $98 \%$ of the volume from the planned and delivered treatment, respectively. A positive difference indicated that the delivered dose was higher than the planned dose, while a negative value indicated that the cumulated delivered dose was lower than the planned dose.

\section{Results}

One of the fifteen patients screened in 7 months, was excluded from the analysis since an excessive variation of the rectum and bladder filling required repeated $\mathrm{CBCT}$ scans before almost each treatment session. The dose calculated on the СВСТ0 gave more than $5 \%$ difference for rectum and bladder ( $\mathrm{V}_{40 \mathrm{~Gy}}$ and $\mathrm{V}_{70 \mathrm{~Gy}}$, respectively) when compared with the one calculated on the planning CT.

The $\mathrm{D}_{98 \%}$ dose value for the PTV, resulting from the DVH of the delivered and planned treatment, is reported for each patient in column two of Table 3. Three of the fourteen patients (patients 3, 4 and 5) did not meet the criterion for PTV coverage, ending the treatment with 
Table 3 The Dose received by $98 \%$ of prostate volume $\left(D_{98 \%}\right)$ at the end of the delivered treatment is reported in column 2, DVHs cut-off points $V_{70 G y}$ for bladder in column 3 , and $V_{40 G y}$ and $V_{70 G y}$ for rectum in columns 4 and 5 respectively; the first value shown is for the planned treatment and in brackets for the delivered one

\begin{tabular}{lllll}
\hline Patient & PTV, $D_{98 \%}$ & $\begin{array}{l}\text { Bladder, } V_{70 G y} \\
D_{98 \%}(G y)\end{array}$ & $\begin{array}{l}\text { Rectum, } V_{40 G y} \\
\text { Vol (\%) }\end{array}$ & $\begin{array}{l}\text { Rectum, } \\
\text { Vol (\%) }\end{array}$ \\
\hline 1 & $69.5(70.2)$ & $23 \%(25 \%)$ & $30 \%(30 \%)$ & $5 \%(4 \%)$ \\
2 & $71.5(72.0)$ & $38 \%(32 \%)$ & $22 \%(22 \%)$ & $2 \%(2.0 \%)$ \\
$3^{\text {a }}$ & $69.0(\mathbf{6 7 . 4 )}$ & $26 \%(26 \%)$ & $51 \%(49 \%)$ & $16 \%(10 \%)$ \\
4 & $71.5(\mathbf{6 6 . 0})$ & $28 \%(36 \%)$ & $26 \%(26 \%)$ & $3 \%(3 \%)$ \\
5 & $71.8(67.0)$ & $15 \%(28 \%)$ & $32 \%(35 \%)$ & $0 \%(0.0 \%)$ \\
6 & $71.6(72.5)$ & $25 \%(3 \%)$ & $27 \%(24 \%)$ & $1 \%(1 \%)$ \\
7 & $70.6(69.8)$ & $30 \%(30 \%)$ & $21 \%(20 \%)$ & $3 \%(2 \%)$ \\
8 & $70.0(71.5)$ & $23 \%(29 \%)$ & $26 \%(25 \%)$ & $3 \%(\%)$ \\
9 & $69.8(70.0)$ & $10 \%(12 \%)$ & $13 \%(32 \%)$ & $0 \%(0 \%)$ \\
10 & $70.7(69.5)$ & $25 \%(25 \%)$ & $12 \%(11 \%)$ & $1 \%(2 \%)$ \\
11 & $69.5(68.5)$ & $24 \%(37 \%)$ & $20 \%(32 \%)$ & $3 \%(4 \%)$ \\
12 & $70.2(69.5)$ & $20 \%(18 \%)$ & $32 \%(32 \%)$ & $0 \%(0 \%)$ \\
13 & $70.0(69.2)$ & $15 \%(10 \%)$ & $55 \%(55 \%)$ & $3 \%(1 \%)$ \\
14 & $70.5(69.7)$ & $23 \%(29 \%)$ & $48 \%(40 \%)$ & $7 \%(5 \%)$ \\
\hline
\end{tabular}

${ }^{a}$ For Patient $3, V_{50 G y}=47 \%$ (79\%) planned and delivered respectively Values out of the expected values ( $2.5 \%$ of difference for $D_{98 \%}$, out of the limits for OARs) are reported in bold

$\mathrm{D}_{98 \%}$ lower than the prescribed dose of about 3.8, 5.7, and $4.2 \%$ respectively. The percentage dose difference for prostate coverage between the planned and delivered treatment is shown in Fig. 1. As can be observed, two of the three patients who ended their treatment with $\mathrm{D}_{98 \%}$ value out of the level of tolerance had already shown a trend towards under-dosing from the earliest fraction (fraction 5 for patients 4 and 5), whereas the underdosing of patient 3 was harder to identify as the patient already started with a low value for $\mathrm{D}_{98 \%}$ (69 Gy) and ended with a percentage difference of $2.3 \%$ (67.4 Gy).

The $D_{\max }$ values obtained from the delivered treatments were comparable to those for the planned treatment: the difference between the maximum planned and delivered dose gave a median value of $2.15 \pm 0.79$ Gy maximum and minimum differences of $3.0 \mathrm{~Gy}$ and 0.5 Gy, respectively. These differences in the $\mathrm{D}_{\max }$ absolute value were considered acceptable by the RO.

Concerning the OARs, the RO identified $\mathrm{V}_{40 \text { Gy }}$ for the rectum and $V_{70 G y}$ for the bladder as the most significant DVH cut-off points for tracking during treatment; this was chosen because their initial values were the closest to the respective limit and could potentially exceed it. $\mathrm{V}_{\text {70Gy }}$ for rectum is a strong predictor of rectal toxicity and was monitored despite the fact that the values obtained were far from exceeding the limit. The results of the clinical optimisation are reported in Table 3, columns 3,4 and 5 . The values obtained at the end of the accumulated delivered treatment are shown in brackets. Two of the fifteen patients failed to meet the bladder constraints (patients 4 and 11), while all patients were within the rectum constraints by the end of treatment. For patient 4 , the $\mathrm{V}_{70 \text { Gy }}$ value for the bladder increased from 28 to $36 \%$, so this patient failed to meet two criteria (i.e., the target coverage and the bladder constraints). For patient 11, the $\mathrm{V}_{70 \mathrm{~Gy}}$ for the bladder rose from 24 to $37 \%$. Although patient 3 first met the cut-off parameter selected by the $\mathrm{RO}$ for the bladder $\left(\mathrm{V}_{70 \mathrm{~Gy}}\right)$, $79 \%$ of his bladder volume unfortunately received a dose of $50 \mathrm{~Gy}$ at the end of the treatment, so he failed both the target dose coverage and the bladder constraint; Fig. 2 shows the comparison of the DVHs for the planned and delivered treatment in this patient.

The cumulative dose delivered to $35 \%$ of the bladder volume $\left(D_{35 \%}\right)$ and $60 \%$ of the rectum volume $\left(D_{60 \%}\right)$ vs

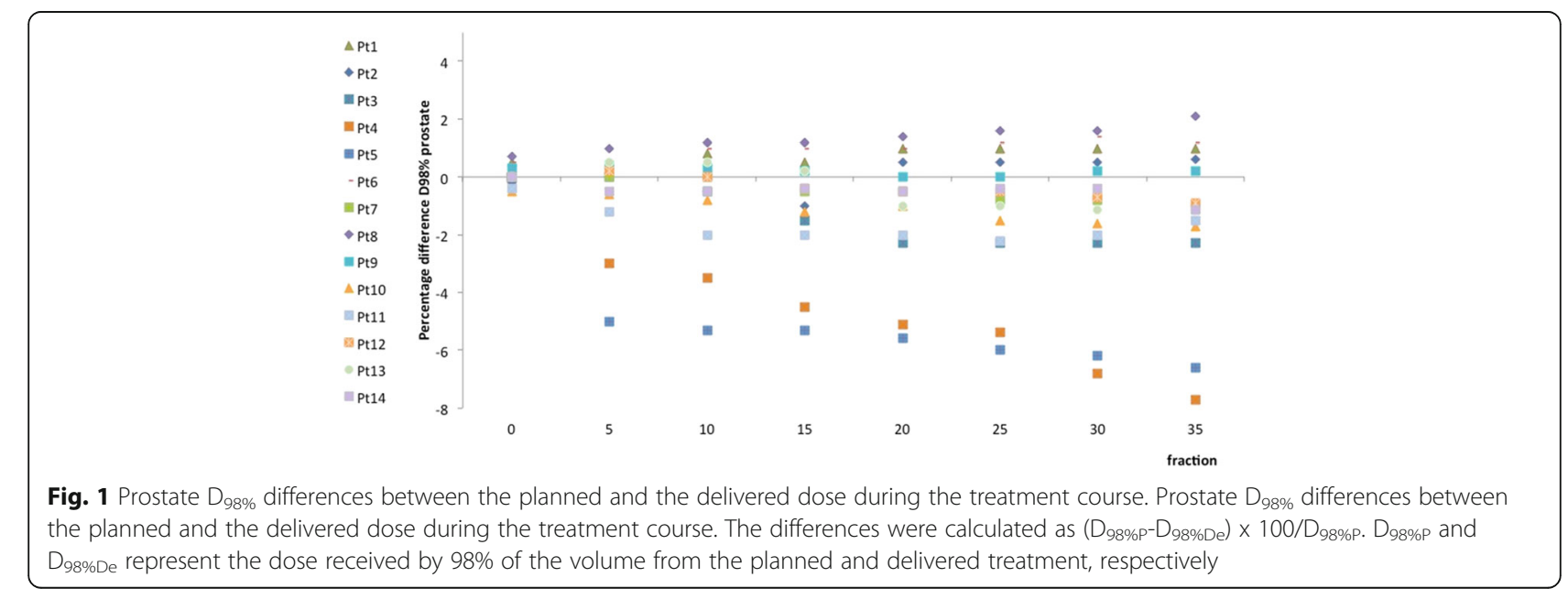




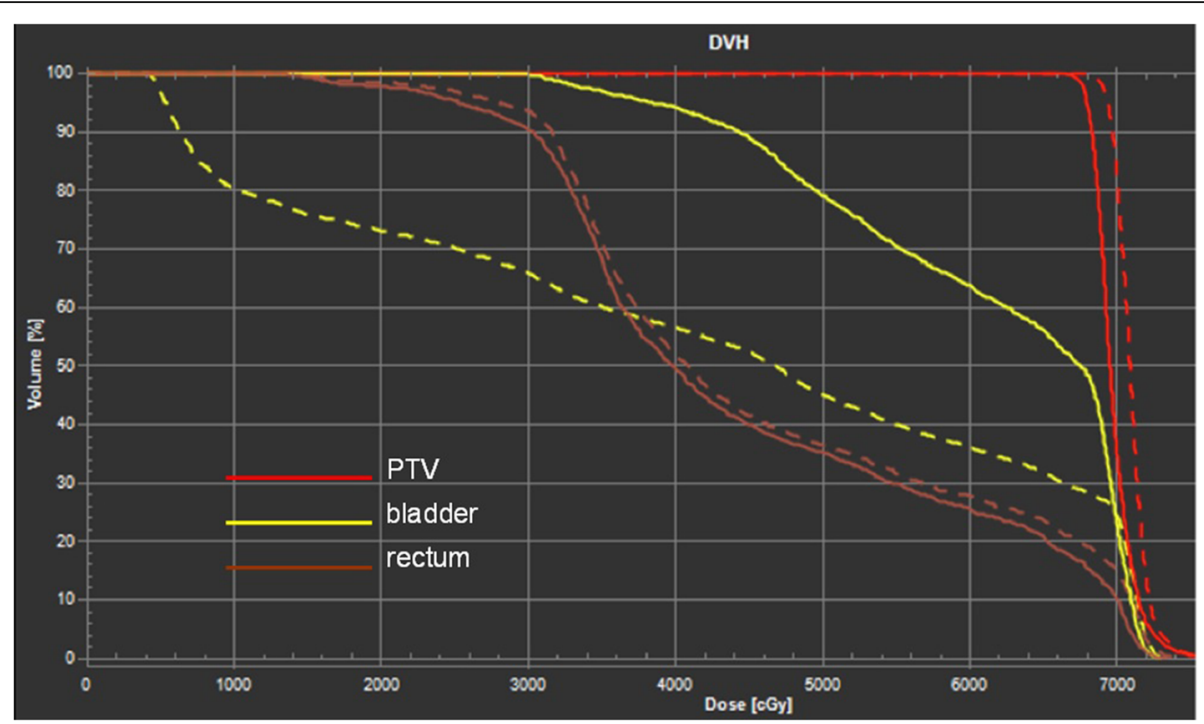

Fig. 2 DVHs comparison of the delivered dose and planned dose for patient 3. DVHs comparison of the delivered dose (solid line) and planned dose (dashed lines) for patient 3. The curves for bladder, rectum and planning target volume (PTV) are reported with yellow, brown and red lines respectively

the treatment fractions for every patient of the study, were reported in Fig. 3a, and b respectively; the solid lines indicate the cut-off values for the rectum and the bladder $\left(\mathrm{D}_{60 \%}<40\right.$ Gy and $\mathrm{D}_{35 \%}<70$ Gy, respectively). Regarding the overall pass criteria (minimum required
PTV coverage and within the OAR constraints), four of fourteen patients did not satisfy one or more criteria by the end of their radiotherapy.

The time needed for the dose tracking procedure averaged $2 \mathrm{~h}$ for the $\mathrm{RO}$ and $4 \mathrm{~h}$ for the medical physicist, with

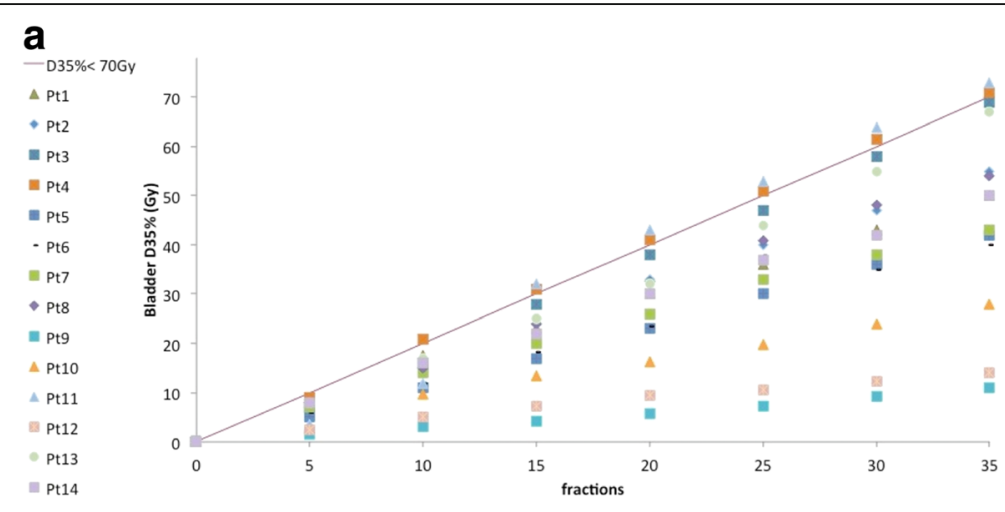

b

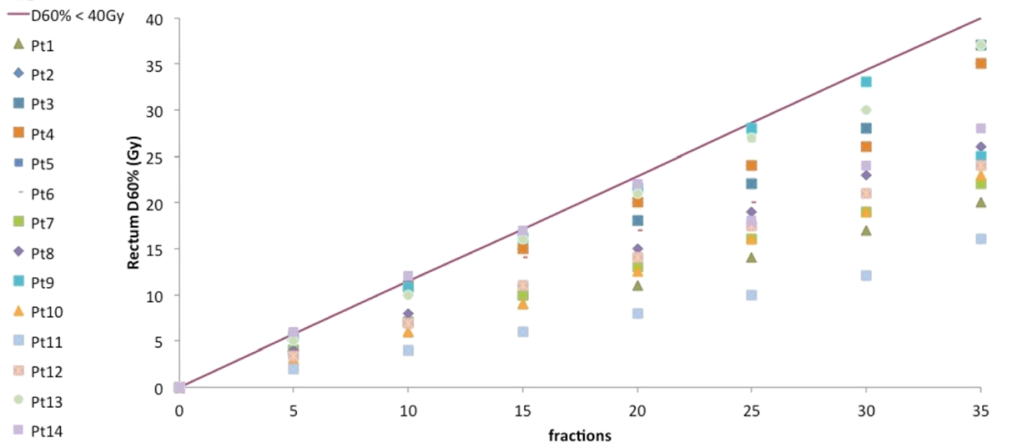

Fig. 3 Evaluation of the cumulative dose delivered to the rectum and to the bladder. The cumulative dose received by $35 \%$ of the bladder volume $\left(D_{35 \%}\right)$ and the dose received by $60 \%$ of the rectum volume $\left(D_{60 \%}\right)$ is reported vs. the treatment fractions in $\mathbf{a}$ and $\mathbf{b}$ respectively. The solid line represents the cut off value for the rectum $\left(D_{60 \%}<40 \mathrm{~Gy}\right)$ and for the bladder $\left(D_{35 \%}<70 \mathrm{~Gy}\right)$ 
no extra time being needed by the therapist (they were not directly involved). In particular, it took about $15 \mathrm{~min}$ for the $\mathrm{RO}$ to complete each $\mathrm{CBCT}$, including the time needed for assessment of the rigid registration, adjustment and assessment of the contours after mapping regions of interest, and final assessment of the deformable registration. By contrast, it required the medical physicist approximately $4 \mathrm{~h}$ to reconstruct and evaluate the cumulative dose. The commonly accepted IGRT procedure required a maximum time of $10 \mathrm{~min}$ for each $\mathrm{CBCT}-\mathrm{CT}$ online matching assessment for the RO (if the therapist called him at the beginning of the CBCT procedure) and for the therapist, while no extra time to the workflow of the medical physicist.

\section{Discussion}

The low image contrast of the MV CBCT can appear a limitation if compared with the $\mathrm{kV}-\mathrm{CBCT}$ although the results of our study were not affected. Other authors dealing with MV-CBCT in clinical situations applied and successfully used a similar protocol $[16,21,28]$. Our ROs, based on their clinical experience, verified that the image quality of $8 \mathrm{MU}$ CBCT protocol adopted was adequate for soft tissue visualization nor influence the registration process with the $\mathrm{kV}$ planning CT.

The acceptance criterion of $\mathrm{D}_{98 \%}>97.5 \%$ for the PTV corresponds to an acceptable difference of 1.7 Gy in the delivered and planned dose. Considering that the prescription was 70 Gy we expected $D_{98 \%}$ dose values $>68.3 \mathrm{~Gy}$. However, three patients did not meet their respective criteria (patients 3, 4, and 5). The PTV was used as the main target by our radiation oncologist throughout treatment, as indicated in Prescribing, Recording and Reporting Photon Beam Therapy (Report 62) by the International Commission on Radiation Units \& Measurements (ICRU) [29], which states that coverage must be in a range between $95 \%$ and $107 \%$ of the prescribed dose.

For the treatment of prostate cancer, we tend to achieve better results than the ICRU recommendation and the $\mathrm{D}_{98 \%}$ parameter is used. However, variation in the organs surrounding the prostate bed may easily lead to the target parameters not being met. Neither patient 3 not patient 4 met the bladder constraint criterion, with patient 4 showing an increase in the $\mathrm{V}_{70 \mathrm{~Gy}}$ value for the bladder from 28 to $36 \%$, and patient 3 showing an increase in $D_{50 G y}$ from 47 to $79 \%$. Even though this was not the initial cut-off point selected to track the treatment, it cannot be ignored. As extensively reported in the literature, surrounding organs may present significant day-to-day variations in shape if they remain uncontrolled during routine treatment. As reported by Frank [4], most patients do not have a full bladder at the time of treatment because this can be difficult to maintain. Even if our patients followed strict rules for treatment preparation $(500 \mathrm{~mL}$ of water $30 \mathrm{~min}$ before treatment and defecation to ensure a full bladder and an empty rectum, respectively), some may still experience difficulties. The use of rectal balloons can help in prostate immobilization and rectal toxicity reduction, and its use is highly investigated and discussed [30]. However, the technical difficulty of placing an endorectal balloon on a daily basis, as well as the patient discomfort associated with this procedure has been weighed against the benefit of the use of this device in our center.

As can be clearly seen in Fig. 3a, the acceptable bladder values (represented by the solid line) had been exceeded by fractions 10 and 15 in patients 4 and 11, respectively. For these two patients, the discrepancies were already present during verification, before treatment and were ascribed to poor compliance in maintaining bladder volume. Despite this discrepancy noted during CBCT0 imaging the patients were enrolled as the patient criteria selection was not affected. It is likely that patient 3 underwent the first CT planning with a full bladder, but was not able to reproduce it again. This different organ configuration may have led to over-dosing of the bladder and an under-dosing of the prostate because of changes in the location of the organs from treatment planning. These results can be extrapolated out to the last intended fraction to help the clinicians to assess if the original treatment goals would be achieved. The final treatment outcomes may have been improved with a replanning procedure during treatment. Different authors $[10,11,31,32]$ analyzed the impact of an adaptive procedure in a clinical treatment, and demonstrated the effectiveness of offline dose compensation technique in IGRT prostate cancer. A single re-planning performed when the dose accumulation process exceeds the clinician thresholds may be necessary during the course of radiotherapy to remediate timely a deviation of the delivered treatment from the planned one. The time of the replanning may be hard to identify, and therefore an optimised strategy of dose tracking must be set up. An early identification of OARs and cut-off points for acceptable upper dose limits, the set-up of the level of intervention (clinician thresholds for altering the planned treatment), and a reasonable frequency of the dose accumulation process, are the main issues discussed in our study. The additional time needed for dose tracking assessment was considered reasonable and less than that needed for an IGRT procedure, resulting in an acceptable impact on workflow in the radiotherapy department.

There are many important and practical concerns to be addressed for a successful dose tracking procedure, for example, intrafraction motion, organ contouring, the accuracy of the image registration and the reliability of deformable organ registration. Currently, many researchers are being carried out to manage these uncertainties, which 
are beyond the scope of this study. The results of our research must be understood within the insightful limitation of this study. The patients were enrolled only if no significant systematic error was found during the CBCT0 imaging; therefore, the results cannot be generalized to the entire population of men. The study involved a limited number of patients coming from a single institution, therefore it can be considered a small study and the contouring even if performed by the same $\mathrm{RO}$ following international guidelines was not validated by multi-institutional quality assurance program. However, the results show that deviations from the initial conditions can arise and that these deviations can lead to dosimetric differences over the target and OARs that cannot be neglected.

The intention of the authors is to go on with the study, investigating how the time of re-planning following the dose tracking procedure proposed can affect the comparison of normal tissue control probability and tumour control probability of the planned and delivered treatments.

\section{Conclusions}

In this group of fourteen patients undergoing adjuvant radiotherapy for prostate cancer, comparison of the $\mathrm{cu}$ mulative dose delivered to the patient and the approved planning dose showed that there was a deviation from the accepted initial conditions of the PTV coverage, the OAR constraints, or both in four patients $(>28 \%)$. In these cases, a re-planning during the course of treatment may have avoided these discrepancies. We conclude supporting the utility and feasibility of dose tracking assessment in a radiotherapy routine as it offers objectives information to support radiation oncologists in making decisions about adaptive procedures with an acceptable added workload.

\section{Abbreviations}

CBCT: Cone beam computed tomography; CT: Computed tomography; CTV: Clinical target volume; DVH: Dose volume histogram; IGRT: Imageguided radiotherapy; IMRT: Intensity-modulated radiotherapy; MR: Magnetic resonance; MV-CBCT: Megavoltage computed tomography; OARs: Organs at risk; PTV: Planning target volume; RO: Radiation oncologist

\section{Acknowledgements \\ None. \\ Funding \\ The authors did not receive any funding for this work.}

\section{Availability of data and materials}

The data are fully available without restriction. The treatments planning, the $\mathrm{CBCT}$ processed and their elaborations were archived in the informatic System of Centro Oncologico Fiorentino.

\section{Authors' contribution}

Design of the Research: LCO, LC; Evaluation of the Images: LC, MC, LCE; Elaboration of the data: LCO, CF; Manuscript Preparation: WP, LCO; Writing Manuscript LCO, CF, WP. All authors read and approved the final manuscript.

\section{Competing interests}

The authors declare that they have no competing interests.

\section{Consent for publication}

Even if no individual patient data were reported, consensus has been received by every patient for the elaboration of its data and for a future scientific publication.

Ethics approval and consent to participate

The authors declare that he retrospective study "Dose tracking assessment for image-guided radiotherapy of the prostate and the impact on clinical workflow" started in April 2015 received the approval of the ethic Committee of the Hospital Centro Oncologico Fiorentino located in Via Attilio Ragionieri 1, Sesto Fiorentino Florence (Italy), where the data evaluations were performed.

\section{Publisher's Note}

Springer Nature remains neutral with regard to jurisdictional claims in published maps and institutional affiliations.

\section{Author details}

${ }^{1}$ Centro Oncologico Fiorentino, Radiation Oncology Department, Via A. Ragionieri 101, 50023 Florence, Italy. ${ }^{2}$ Department of Radiation Oncology, Sichuan Cancer Hospital, No.55, the 4th Section, Renmin South Road, Chengdu, China. ${ }^{3}$ Azienda Ospedaliera di Perugia, Health Physics Department, Piazzale Menghini 1, 06129 Perugia, Italy.

Received: 6 February 2017 Accepted: 25 April 2017

Published online: 28 April 2017

\section{References}

1. Bauman G, Rumble RB, Chen J, Loblaw A, Warde P. Intensity-modulated radiotherapy in the treatment of prostate cancer. Clin Oncol. 2012;24(7): 461-73

2. Kame'enui EJ, Simmons DC. Knowledge-based IMRT treatment planning for prostate cancer. Med Phys. 2011;38(5):2515-22.

3. Veldeman L, Madani I, Hulstaert F, De MG, Mareel M, De NW. Evidence behind use of intensity-modulated radiotherapy: a systematic review of comparative clinical studies. Lancet Oncol. 2008;9(4):367.

4. Frank SJ, Dong L, Kudchadker RJ, De CR, Lee AK, Cheung R, et al. Quantification of prostate and seminal vesicle interfraction variation during IMRT. Int J Radiat Oncol Biol Phys. 2008;71(3):813.

5. Hoogeman MS, Van HM, De BJ, Lebesque JV. Strategies to reduce the systematic error due to tumor and rectum motion in radiotherapy of prostate cancer. Radiother Oncol. 2005;74(2):177-85.

6. Kupelian PA, Langen KM, Willoughby TR, Wagner TH, Zeidan OA, Meeks SL. Daily variations in the position of the prostate bed in patients with prostate cancer receiving postoperative external beam radiation therapy. Int J Radiat Oncol Biol Phys. 2006;66(2):593-6.

7. Reddy NM, Nori D, Chang H, Lange CS, Ravi A. Prostate and seminal vesicle volume based consideration of prostate cancer patients for treatment with 3D-conformal or intensity-modulated radiation therapy. Med Phys. 2010; 37(7):3791-801.

8. Bylund KC, Bayouth JE, Smith MC, Hass AC, Bhatia SK, Buatti JM. Analysis of interfraction prostate motion using megavoltage cone beam computed tomography. Int J Radiat Oncol Biol Phys. 2008;72(3):949-56.

9. Kupelian P, Meyer JL. Image-guided, adaptive radiotherapy of prostate cancer: toward New standards of radiotherapy practice. Front Radiat Ther Oncol. 2011:43:344-68.

10. Ghilezan M, Yan D, Martinez A. Adaptive radiation therapy for prostate cancer. Semin Radiat Oncol. 2010;20(2):130-7.

11. Han Liu QW. Evaluations of an adaptive planning technique incorporating dose feedback in image-guided radiotherapy of prostate cancer. Med Phys. 2011;38(12):6362-70.

12. Nuver TT, Hoogeman MS, Remeijer P, Van HM, Lebesque JV. An adaptive off-line procedure for radiotherapy of prostate cancer. Int J Radiat Oncol Biol Phys. 2007;67(5):1559-67.

13. Wu Q, Liang J, Yan D. Application of dose compensation in image-guided radiotherapy of prostate cancer. Phys Med Biol. 2006;51(6):1405-19.

14. Michalski JM, Lawton C, El NI, Ritter M, O'Meara E, Seider MJ, et al. Development of RTOG consensus guidelines for the definition of the clinical 
target volume for postoperative conformal radiation therapy for prostate cancer. Int J Radiat Oncol Biol Phys. 2010;76(2):361-8.

15. Johnson M. Patient dose and image quality from mega-voltage cone beam computed tomography imaging. Med Phys. 2007;34(2):499-506

16. Morin O, Gillis A, Descovich M, Chen J, Aubin M, Aubry JF, et al. Patient dose considerations for routine megavoltage cone-beam CT imaging. Med Phys. 2007;34(5):1819-27.

17. Miften M, Gayou O, Reitz B, Fuhrer R, Leicher B, Parda DS. IMRT planning and delivery incorporating daily dose from mega-voltage cone-beam computed tomography imaging. Med Phys. 2007;34(10):3760-7.

18. Hodapp N. The ICRU Report 83: prescribing, recording and reporting photon-beam intensity-modulated radiation therapy (IMRT). Strahlenther Onkol. 2012;188(1):97.

19. Jackson A, Marks LB, Bentzen SM, Eisbruch A, Yorke ED, Ten Haken RK, et al. The lessons of QUANTEC: recommendations for reporting and gathering data on dose-volume dependencies of treatment outcome. Int J Radiat Oncol Biol Phys. 2010;76(3):155-60.

20. Lee WR, Dignam JJ, Amin M, Bruner D, Low D, Swanson GP, et al. NRG Oncology RTOG 0415: A randomized phase III non-inferiority study comparing two fractionation schedules in patients with low-risk prostate cancer. Int J Radiat Oncol Biol Phys. 2016;94(1):3-4.

21. Morin O, Gillis A, Chen J, Aubin M, Bucci MK, lii MR, et al. Megavoltage cone-beam CT: System description and clinical applications. Med Dosim. 2006;31(1):51-61.

22. García-Mollá R, De M-BN, Bonaque J, Vidueira L, López-Tarjuelo J, PerezCalatayud J. Validation of a deformable image registration produced by a commercial treatment planning system in head and neck. Phys Med. 2015;31(3):219-23.

23. Hardcastle N, Elmpt WW, Ruysscher DD, Bzdusek K, Tomé WA. Accuracy of deformable image registration for contour propagation in adaptive lung radiotherapy. Radiat Oncol. 2013;8(1):1-8.

24. Sarrut D. Deformable registration for image-guided radiation therapy. Zeitschrift Für Medizinische Physik. 2006;16(4):285.

25. Schreibmann E, Pantalone P, Waller A, Fox T. A measure to evaluate deformable registration fields in clinical settings. J Appl Clin Med Phys. 2012;13(5):3829.

26. Mzenda B, Mugabe KV, Sims R, Godwin G, Loria D. Modeling and dosimetric performance evaluation of the RayStation treatment planning system. J Appl Clin Med Phys. 2014;15(5):29-46.

27. Weistrand O, Svensson S. The ANACONDA algorithm for deformable image registration in radiotherapy. Med Phys. 2015;42:40.

28. Sumida I. Megavoltage cone beam computed tomography dose and the necessity of reoptimization for imaging dose-integrated intensitymodulated radiotherapy for prostate cancer. Int I Radiat Oncol Biol Phys. 2012;82(5):1715.

29. Morgan-Fletcher SL. Prescribing, recording and reporting photon beam therapy (supplement to ICRU report 50), ICRU report 62. ICRU, pp. ix + 52, 1999 (ICRU Bethesda, MD). Br J Radiol. 2001;74(879):294.

30. Teh BS, Mcgary JE, Dong L, Mai WY, Carpenter LS, Lu HH, et al. The use of rectal balloon during the delivery of intensity modulated radiotherapy (IMRT) for prostate cancer: more than just a prostate gland immobilization device? Cancer J. 2002;8(6):476-83.

31. Qin A, Sun Y, Liang J, Yan D. Evaluation of online/offline image guidance/ adaptation approaches for prostate cancer radiation therapy. Int J Radiat Oncol Biol Phys. 2015;91(5):1026.

32. Stanley K, Eade T, Kneebone A, Booth JT. Investigation of an adaptive treatment regime for prostate radiation therapy. Pract Radiat Oncol. 2014;5(1):e23-e9.

\section{Submit your next manuscript to BioMed Central and we will help you at every step:}

- We accept pre-submission inquiries

- Our selector tool helps you to find the most relevant journal

- We provide round the clock customer support

- Convenient online submission

- Thorough peer review

- Inclusion in PubMed and all major indexing services

- Maximum visibility for your research

Submit your manuscript at www.biomedcentral.com/submit
Biomed Central 